\title{
Conscience politique et conscience photographique
}

Political consciousness and photographic consciousness

\section{Xavier Nerrière}

\section{OpenEdition}

\section{Journals}

Édition électronique

URL : https://journals.openedition.org/itti/1180

DOI : 10.4000/itti. 1180

\section{Éditeur}

Université de Poitiers

\section{Référence électronique}

Xavier Nerrière, "Conscience politique et conscience photographique », Images du travail, travail des images [En ligne], 2 | 2016, mis en ligne le 01 septembre 2016, consulté le 29 juillet 2021. URL : http:// journals.openedition.org/itti/1180 ; DOl : https://doi.org/10.4000/itti.1180

Ce document a été généré automatiquement le 29 juillet 2021.

Images du travail, travail des images 


\title{
Conscience politique et conscience photographique
}

Political consciousness and photographic consciousness

\author{
Xavier Nerrière
}

1 Les militants, politiques ou syndicaux, ainsi que leurs organisations, accumulent un nombre considérable de photographies. La Loire-Atlantique dispose, avec le Centre d'histoire du travail (CHT), d'une structure originale qui s'est donné pour objet de conserver et de mettre en valeur la mémoire des conflits sociaux dans ce département, quel qu'en soit le support ${ }^{1}$. Parmi la matière accumulée figurent donc naturellement de nombreuses images, environ 50000.

2 Nous nous sommes efforcés, à travers une récente publication (Nerrière, 2014), de dresser une typologie de ces documents. Ce travail d'analyse nous a conduits à échafauder des hypothèses qu'il nous faut maintenant éprouver. En premier lieu nous avons identifié, à partir du corpus conservé par le CHT, trois grandes catégories d'images relatives au monde du travail. Il existe selon nous un premier ensemble que nous pourrions qualifier de "photographies industrielles ». Souvent commanditées par l'employeur, parfois directement réalisées par ses soins ou des membres de l'encadrement, ces images sont très précieuses pour décrire l'appareil productif. Un second groupe pourrait être qualifié de "photographies de presse». Les commanditaires sont alors extérieurs à l'entreprise et cette source permet en particulier de suivre les événements économiques (inaugurations d'entreprises, nouvelles productions...) et sociaux (les conflits). Nous estimons qu'un troisième groupe pourrait être défini par l'expression « auto-représentations ». Il s'agit essentiellement de photographies amateur réalisées par les ouvriers ou les employés sur leur lieu de travail et pendant leur service. Elles nous offrent une description de l'ambiance de l'atelier par celles et ceux qui la vivent ou la subissent. L'auto-représentation ne se substitue pas aux autres types de photographie du monde du travail, elle les complète en exprimant des points de vue qui, par définition, ne peuvent pas être photographiés par quelqu'un d'autre. 
Photographie 1. Le « chantier fer » de l'entreprise Champenois.

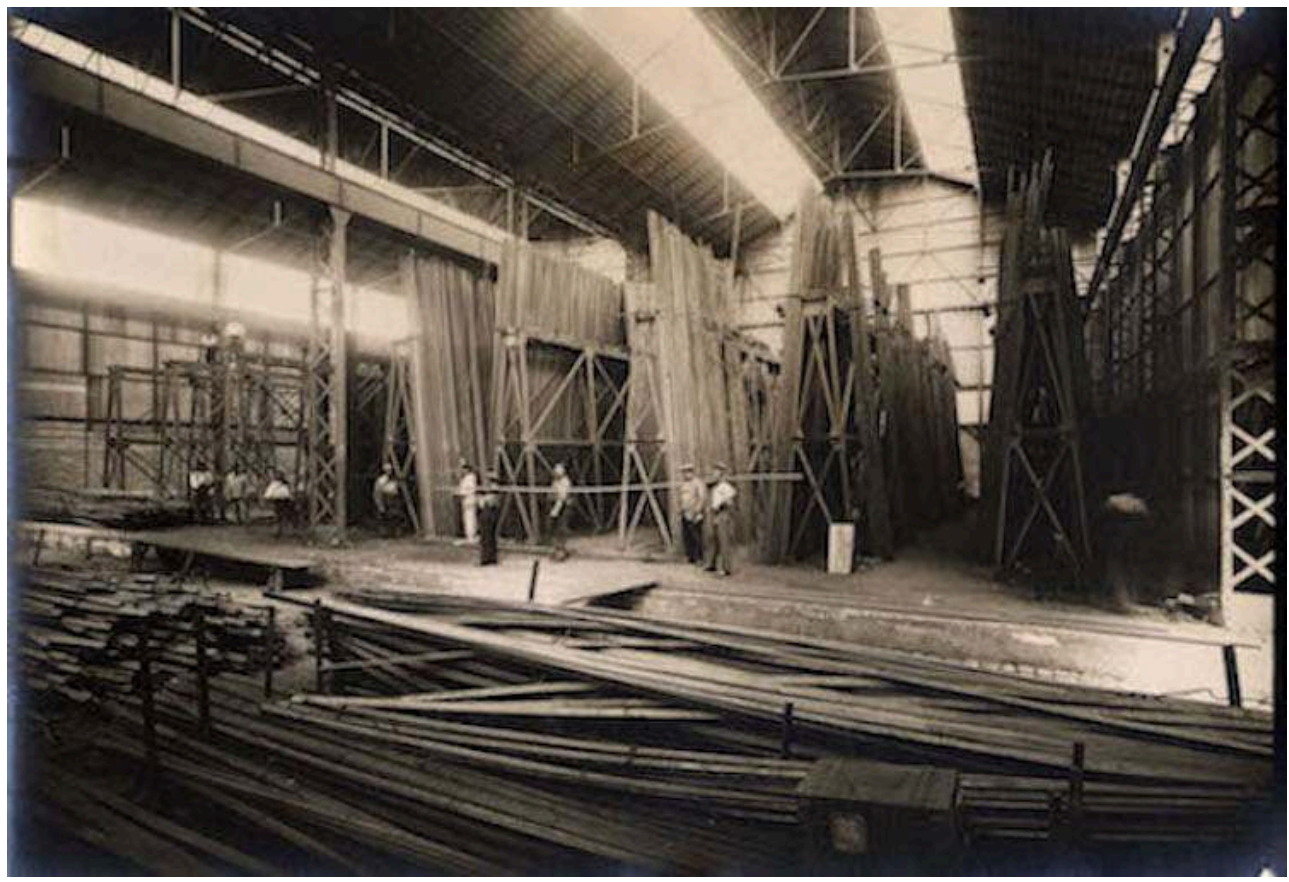

Un exemple de «photographie industrielle », l'intérieur du tout nouveau chantier de fer (ou entrepôt de fer) de l'entreprise Champenois, boulevard de La Prairie-au-Duc à Nantes, le 31 juillet 1923. Le

photographe met en évidence la modernité de l'installation pour l'époque : la propreté, la luminosité, l'agencement des matériaux et les hommes servent en quelque sorte d'échelle pour apprécier les volumes.

$\mathrm{CHT}$, coll. Famille Champenois-Rigault. 
Photographie 2. Grévistes de l'usine Chantelle par une photographe de presse.

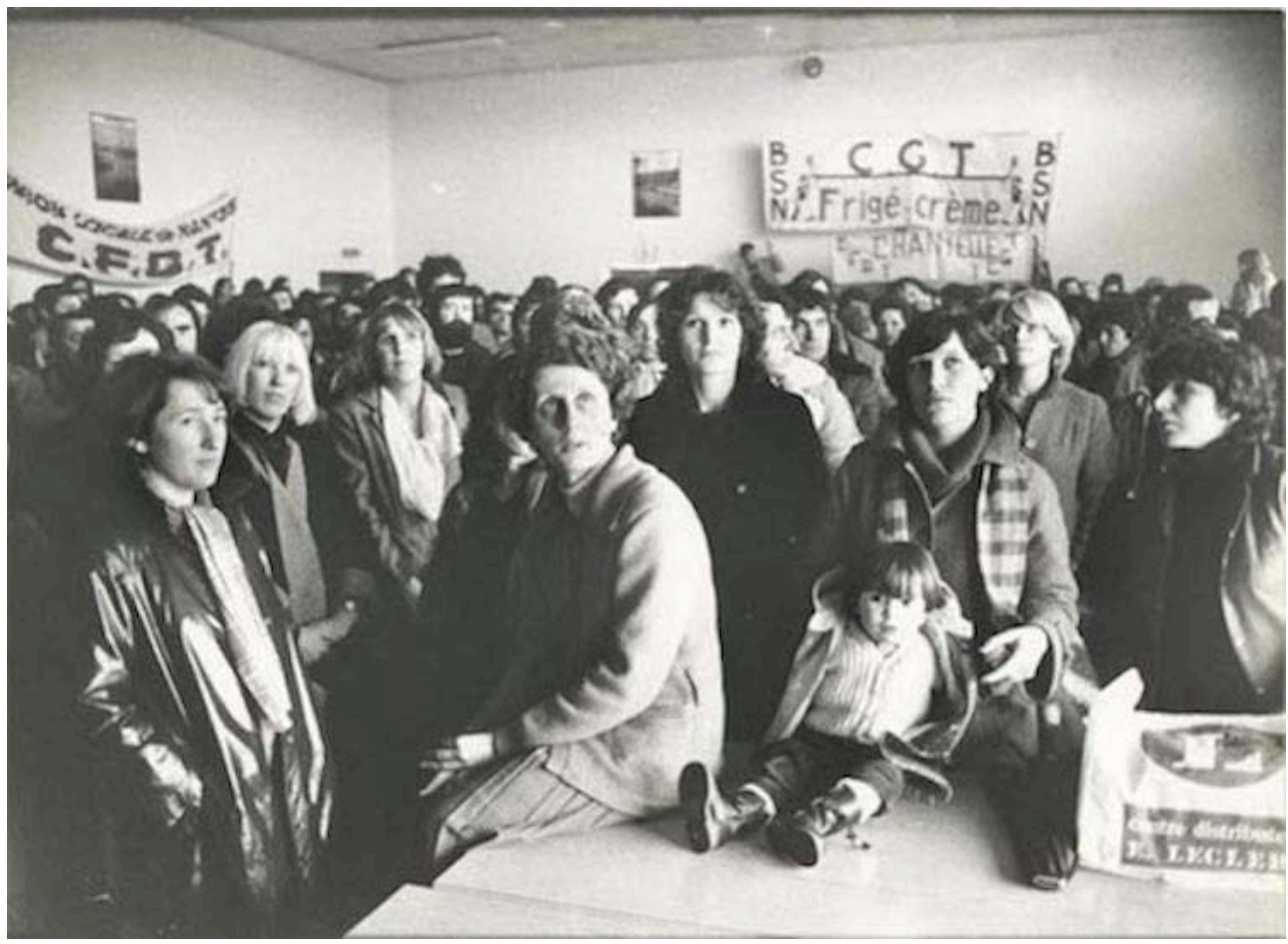

Assemblée de grévistes au sein de l'usine Chantelle, à Saint-Herblain, en décembre 1981. Ce cliché d'Hélène Cayeux, photographe au journal Ouest-France, respecte les exigences de lisibilité d'une "photographie de presse ».

CHT, cliché Hélène Cayeux, coll. UD CGT 44. 
Photographie 3. Un maçon photographié par ses collègues.

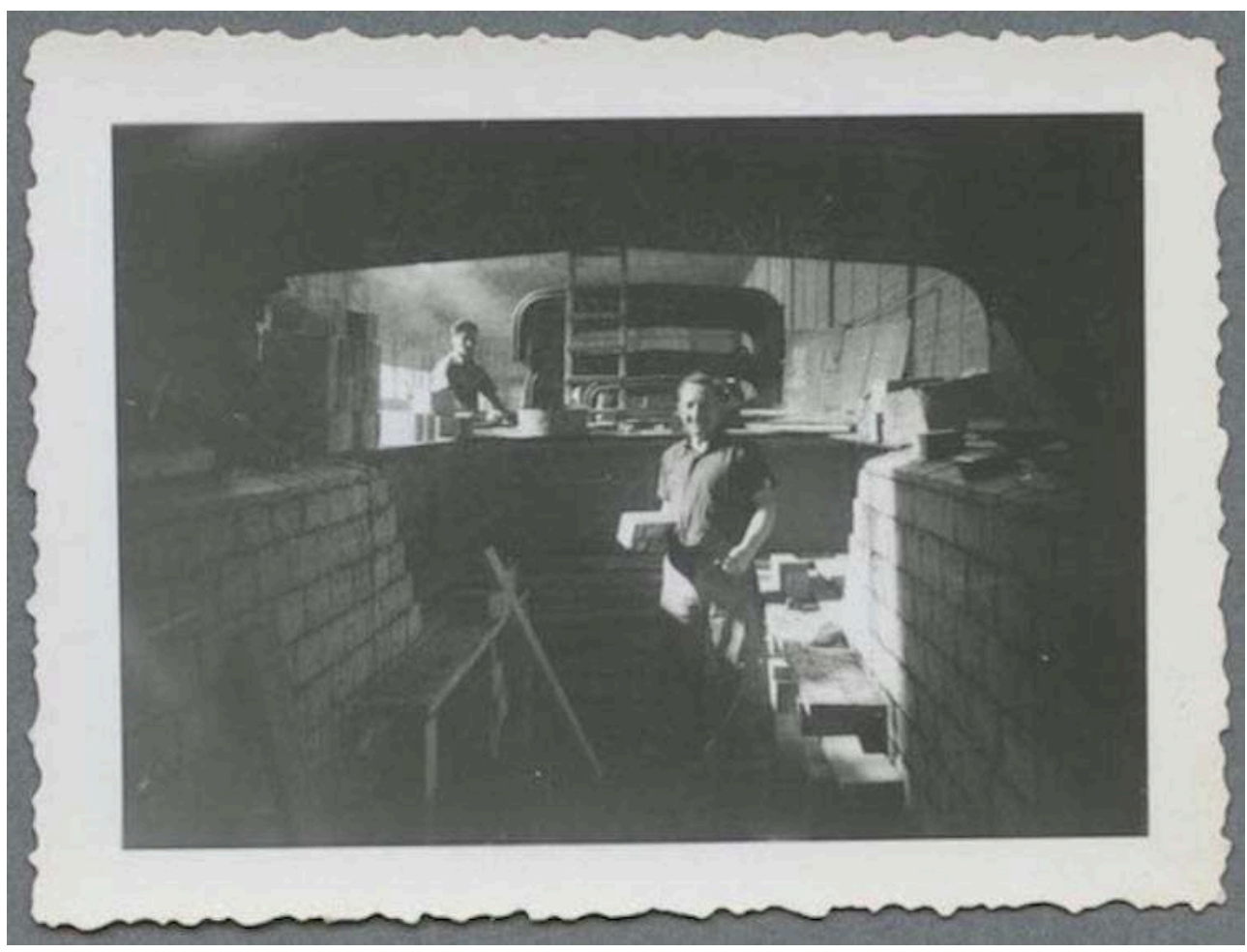

Un ouvrier maçon construit un four, sans doute dans l'entreprise J.-J. Carnaud, ex Forges de BasseIndre, années 1950. Un exemple d'auto-représentation ouvrière déposée au CHT en 2014.

CHT, coll. Jean-Claude Ménard.

Nous écartons volontairement de cette tentative de classification les notions de photographie d'art, ou d'auteur, considérant que ces approches sont transversales aux catégories énoncées : soit l'intervention de l'artiste est sollicitée par l'industriel, ou la direction de l'entreprise, et dans un tel cas les images produites entrent dans la catégorie que nous appelons "photographie industrielle»; soit il intervient de sa propre initiative et sa démarche est proche de celle d'un journaliste (ou d'un documentariste); soit il répond à l'invitation d'instances représentatives du personnel, tel que les Comités d'entreprise (CE), et son travail implique alors souvent le personnel, dans une logique d'éducation populaire, proche cette fois-ci de l'auto-représentation².

En second lieu nous voudrions souligner le fait que chaque image produite sur le monde du travail est potentiellement porteuse d'un discours sur l'entreprise, souvent sensiblement différent suivant les catégories précédemment décrites. La photographie, comme toute science ou technique, n'est pas neutre et nous estimons en particulier que l'analyse des auto-représentations est un moyen d'avoir accès à l'intimité de son auteur avec son univers de travail. Malheureusement, de tels clichés sont théoriquement prohibés puisque l'entreprise est considérée comme une propriété privée. L'employeur, le patron, dispose d'un droit discrétionnaire de réaliser des photographies ou d'autoriser une personne, salariée ou extérieure à l'entreprise, de le faire. Cette contrainte semble largement connue et intégrée par les acteurs eux-mêmes. Cependant, force est de constater que de tels clichés existent et nous n'avons pas à ce jour connaissance de contentieux, prud'homaux ou simplement disciplinaires, relatifs à la réalisation non autorisée de clichés sur un lieu de travail. 
Ces auto-représentations sont difficilement accessibles, souvent enfouies dans les albums de famille. Elles constituent l'une des richesses de la collection conservée par le $\mathrm{CHT}$ et nous avons l'intuition que, vu les précautions que nécessite leur réalisation, elles sont le symptôme d'une forme d'engagement, politique, syndical ou culturel, de la part de leurs auteurs. N'y aurait-il pas une corrélation entre la conscience politique d'un photographe amateur et ce que nous pourrions appeler sa «conscience photographique »? Jean-Louis Davault, un ancien ouvrier de l'arsenal d'Indret (un établissement de la DCNS dépendant du ministère de la Défense) nous a adressé un long courrier où il nous explique : "Je passerai outre ce type de règlement [interdiction de réaliser des photographies à l'intérieur de l'établissement], en 1974, bien décidé à figer un collègue plus âgé - la pâte des hommes - qui m'avait initié aux ficelles de la profession. À l'époque je m'exerçais à peaufiner des portraits [...] Bien entendu, je n'ai pas passé la porte de l'arsenal d'Indret l'appareil photo en bandoulière: il était dissimulé dans mon porte-documents. » Il ajoute : "J'avoue ne jamais avoir fait le lien entre la photographie et l'engagement syndical.» Pourtant, au détour d'une conversation, il affirme à l'inverse mettre certaines photographies en évidence à son domicile pour rappeler à ses petits-enfants « l'origine ouvrière de la famille ».

Photographie 4. Une « équipe moteur » des Ateliers et chantiers de Bretagne (ACB).

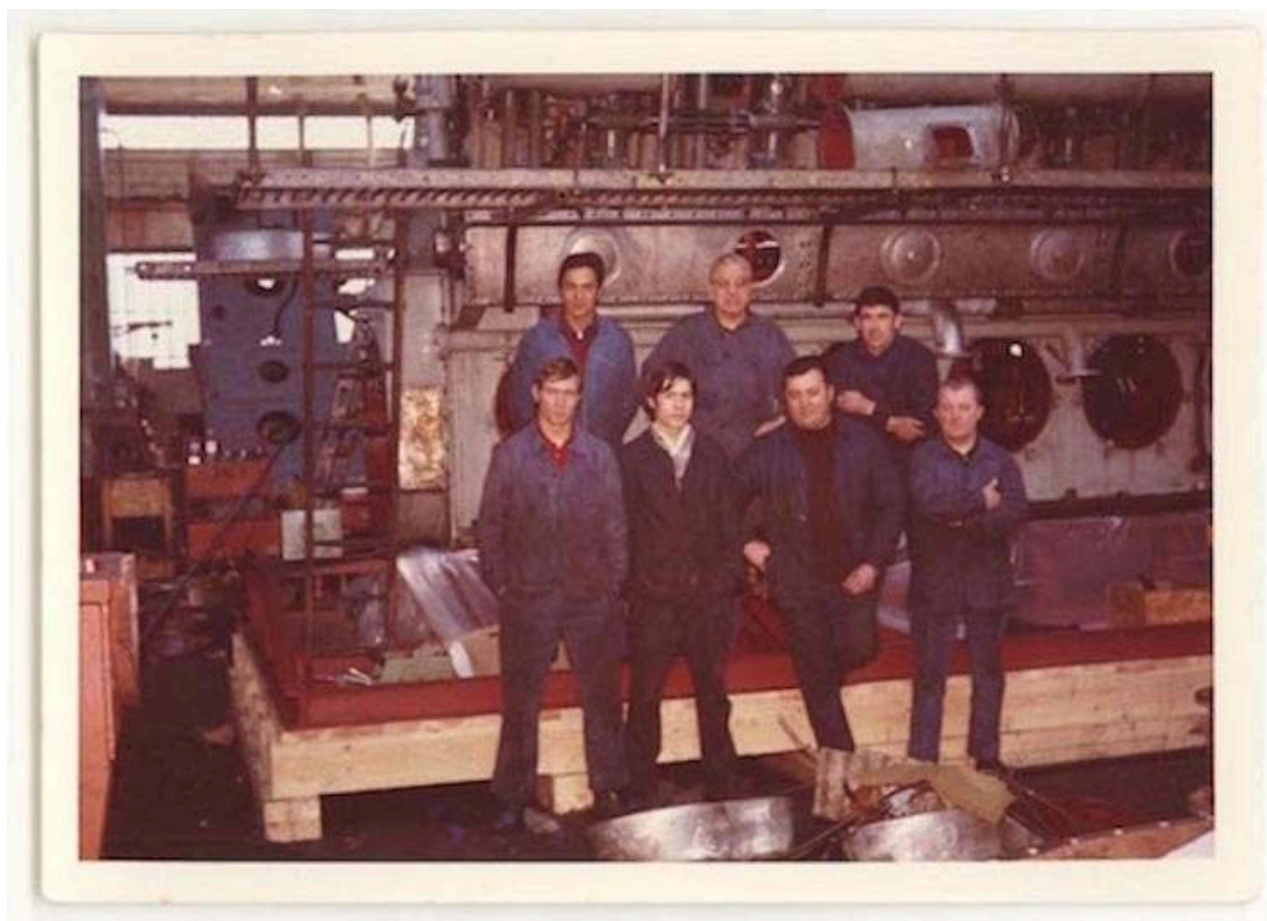

Les membres de l'équipe posent fièrement devant un bâti moteur (la légende précise : « un 6 cylindres en ligne ») destiné à une centrale électrique de Lomé, au Gabon, en 1970. Cette image est conservée par l'un des ouvriers et, à son décès, sa petite-fille la confie au CHT.

$\mathrm{CHT}$, coll. René Bouillant. 
Photographie 5. Photo d'équipe avant le début de la saison touristique.

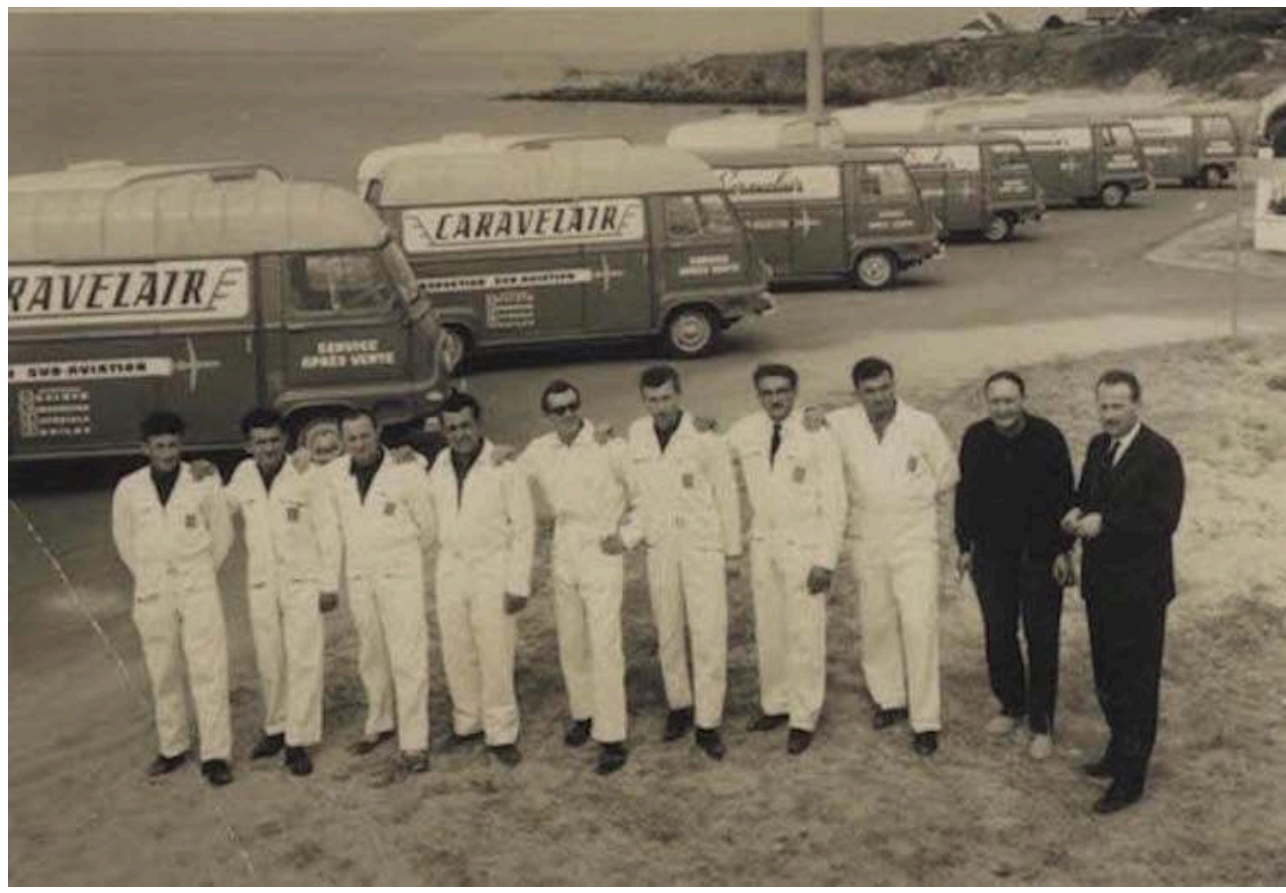

Le service après-vente de la Société européenne de matériels mobiles (SEMM), filiale de l'usine Sudaviation de Saint-Nazaire et productrice des caravanes Caravelair. II s'agit bien, ici, de mettre en scène la communauté de travail unie au sein de l'entreprise, de l'ouvrier aux cadres dirigeants. Nous percevons bien entre ces deux photographies une différence de discours. Pour autant, cette seconde image, certainement le fruit d'une commande de la direction de l'entreprise, a été conservée par la section syndicale CGT.

CHT, coll. CGT SEMM-SOTRIMEC.

6 Face à de telles interrogations et afin d'essayer de confirmer ou d'infirmer les conclusions que nous a inspiré notre travail, nous avons décidé de faire évoluer nos pratiques en matière de collectes iconographiques. Dorénavant, nous essayons de réaliser un entretien avec le déposant, surtout s'il s'agit de l'auteur des clichés, et nous avons dressé une liste de sept thèmes à aborder.

1. Qui a pris les photos?

La réponse n'est pas si évidente, certaines corporations qui bénéficient d'une très forte identité, comme les dockers, pratiquent volontiers l'échange d'images. Dans d'autres cas, le déposant figure sur l'une ou plusieurs des photographies du dépôt. Il ne peut donc pas en être l'auteur, à moins d'avoir eu recours à un dispositif de retardement. Pour finir sur cette première question, la construction des photos (choix de la mise en scène et du lieu de la prise de vue) peut être collective, renvoyant à un mode de fonctionnement «naturel » du groupe concerné. L'auteur ne serait donc plus un individu mais le groupe.

2. Dans quelles circonstances (ou pourquoi) les photos ont-elles été prises?

S'agit-il d'un événement exceptionnel, comme le lancement d'un bateau dans l'industrie navale, un départ en retraite...?

3. Comment la démarche de l'auteur a-t-elle été perçue par ses collègues?

Si l'auteur est seul à prendre l'initiative de la photographie, ses collègues ont-ils eu une réaction différente que si celle-ci avait été le fait d'une personne extérieure au groupe: extérieure à l'entreprise, ou un membre de l'encadrement?

4. À quoi ces photos ont-elles servi?

Était-ce des photos à usage privé, destinées à rester dans un album de famille, ou devaientelles alimenter un fonds syndical, servir à appuyer une revendication en termes de 
conditions de travail... ? Certaines de ces images ont-elles été publiées, sur des tracts, des journaux, ou des réseaux sociaux sur internet?

5. En réalisant telle ou telle prise de vue, l'auteur pensait-il à une autre image plus connue? Autrement dit, quelle est la culture photographique de l'auteur? Quels sont les liens entre photographie populaire (ou vernaculaire) et photographie institutionnelle (artistique) ?

6. L'auteur pratique-t-il souvent la photographie?

Que ce soit dans la sphère familiale ou sur son lieu de travail, quelle est la pratique photographique de l'auteur? A-t-il, tout au long de sa carrière, régulièrement pris des photos sur son lieu de travail ? Quels sont les moments ou les événements qu'il privilégie (ou privilégiait)?

7. Pourquoi a-t-il décidé de confier ses clichés au CHT?

Qu'est-ce qui l'a poussé à réaliser ce dépôt, qu'en attend-t-il, quelle signification donne-t-il à ce dépôt?

7 Ces entretiens ne sont pas standardisés, l'ordre et le nombre des questions peuvent évoluer en fonction des circonstances et des réponses de la personne. L'idée de cette démarche est de profiter d'un poste d'observation privilégié, un centre d'archives syndicales et sociales, une structure militante et patrimoniale, pour accumuler une masse de témoignages permettant peut-être, dans un second temps, de dégager quelques grandes tendances quant au rapport que les classes populaires nourrissent avec leur image, celle de leur travail et de leurs conditions de vie en général. Accessoirement, cette démarche devrait permettre de mieux appréhender la diffusion populaire du médium photographique.

Depuis avril 2014, nous avons eu l'occasion de réaliser plusieurs de ces entretiens et nous vous proposons ici un premier compte-rendu à partir de quatre d'entre eux. Nous avons sélectionné ces personnes parce qu'elles venaient de nous déposer, ou de nous autoriser à reproduire, un nombre conséquent de photographies. Le processus qui conduit à un tel dépôt est souvent long et sinueux, les choses se font par étape. Au départ il y a souvent un projet, par exemple une exposition pour laquelle nous avons besoin de rassembler de la documentation en plus de ce que nous conservons parmi les archives du Centre. Nous sommes ainsi amenés à « découvrir » des collections privées (individuelles ou collectives) de photographies en rapport avec l'histoire sociale. En fonction de l'intérêt des documents mis à jour, nous essayons de convaincre le propriétaire des photos de les déposer, ou d'en autoriser la reproduction, soit par un centre d'archives publiques (Archives municipales ou départementales), soit par le CHT.

\section{Raymond Tessier, un correspondant du journal l'Humanité (entretien réalisé le 17 avril 2014)}

Nous avons été mis en contact avec Raymond Tessier alors que nous travaillions à la réalisation d'un ouvrage intitulé Debout et libre, consacré à une série de conflits au sein de l'usine Aérospatiale de Saint-Nazaire. Raymond Tessier, né en 1935, a été salarié de cette usine de 1954 à 1983, date à laquelle il rejoint un établissement Eurocopter en région parisienne. Parallèlement à son activité professionnelle, Raymond Tessier a été correspondant (bénévole) du journal L'Humanité. Ses clichés, d'une qualité formelle assez remarquable, reviennent souvent lorsque l'on s'intéresse à des conflits sociaux de la région nazairienne impliquant soit la CGT, soit le Parti communiste: « La 
photographie était ma façon de militer ». Les militants de ces deux organisations brandissent ses photos avec fierté, et ce sont eux qui nous ont mis en relation avec Raymond.

Photographie 6. Le photographe se fait le relais de l'humour de ses camarades.

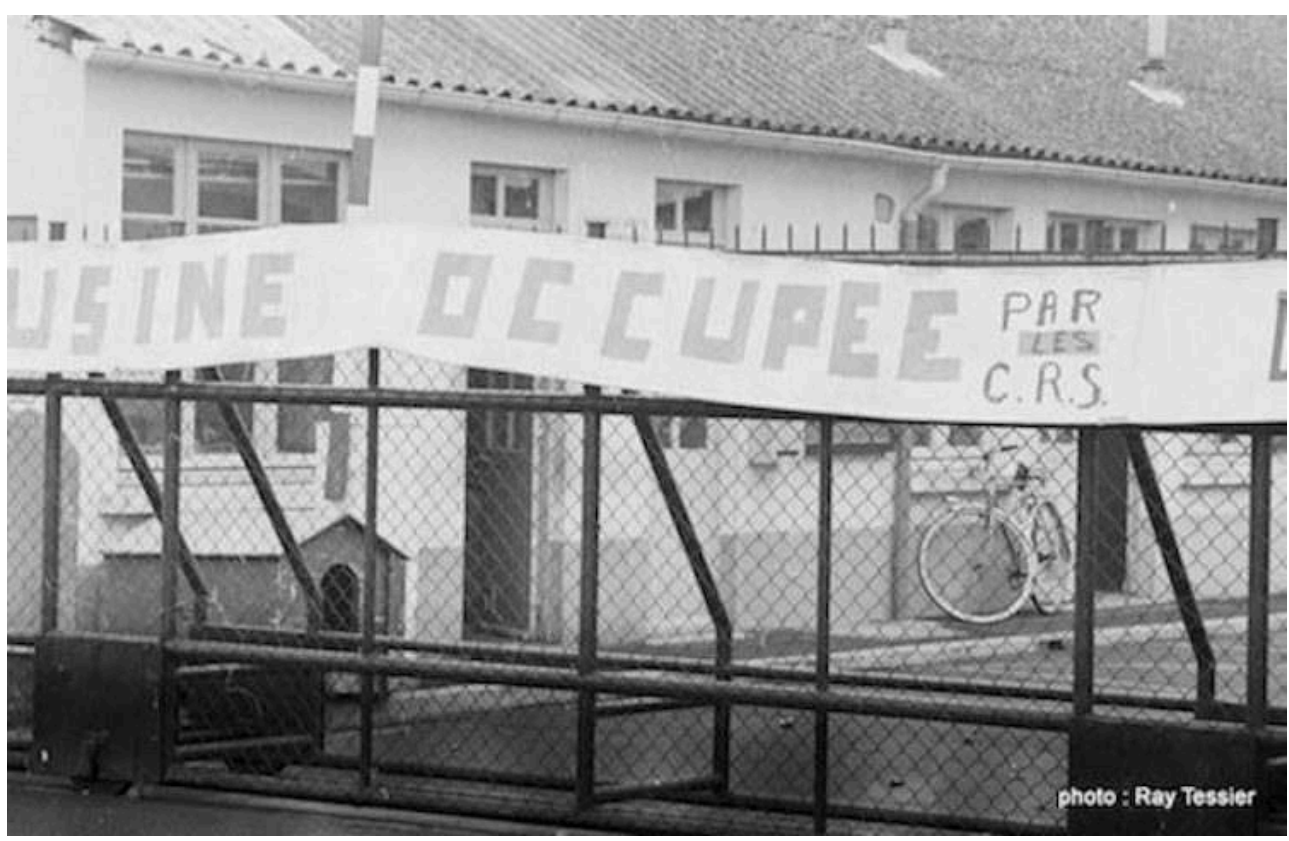

Occupation de l'usine Aérospatiale de Saint-Nazaire par les forces de l'ordre en 1980. L'expression appropriée est en fait lock-out. L'humour et la dérision sont souvent très présents dans les conflits sociaux.

CHT, cliché « Ray Tessier ».

10 Il réalisait ses clichés le matin ou le soir, en dehors de son temps de travail, jamais dans l'entreprise, «c'était interdit ", et il ajoute: "l'Aérospatiale était soumise au secret militaire ». Du coup, il n'a jamais photographié ses copains sur leur lieu de travail comme l'a fait Jean-Louis Davault à l'Arsenal d'Indret. Le parallèle entre ces deux personnes est intéressant, l'un milite (syndicalement et politiquement) à travers sa pratique photographique et respecte scrupuleusement l'interdiction de photographier dans l'usine, sauf lorsque celle-ci est occupée et aux mains des grévistes. L'autre ne fait pas de lien entre auto-représentation photographique et engagement syndical, mais transgresse sciemment l'interdiction d'introduire un appareil photographique dans l'établissement. Encore une fois notre panel est trop étroit pour tirer des conclusions définitives, disons seulement que d'une part Monsieur Davault se situe plus sur un champ culturel (qui n'est potentiellement pas moins subversif), alors que Monsieur Tessier s'inscrit dans une action collective, syndicale et politique. D'autre part, il n'est pas à exclure que les directions modulent l'interdiction de photographier en fonction des circonstances et l'utilisent comme une épée de Damoclès qui leur permettrait de se débarrasser à peu de frais d'un militant trop actif.

11 Raymond a déjà déposé son fonds photographique (30 000 négatifs) auprès des Archives départementales de Seine-Saint-Denis. Il ne propose donc au CHT («pour que ça ne se perde pas ») que deux CD contenant des reproductions numériques de ses clichés, l'un sur la grève dite des mensuels, à Saint-Nazaire en 1967, l'autre sur un conflit dans l'établissement Aérospatiale de Saint-Nazaire en 1979-1980. Mais de tout temps il a 
généreusement distribué ses clichés : aux journaux nationaux de la CGT, aux Nouvelles de Loire-Atlantique, le journal local du Parti communiste, ou aux structures syndicales locales. Et il est fort probable que parmi les tirages marqués «correspondant de l'Huma ", au sein du fonds de l'UD CGT 44 (conservé par le CHT), beaucoup soient de lui.

Sa pratique photographique, il l'a héritée de son père, ouvrier du bâtiment puis dans une usine de matériel agricole. C'est son père qui lui a offert son premier appareil, et à son tour il a transmis sa passion à son fils, "qui fait de meilleures photos que moi ». Nous voyons ici se dessiner une tradition familiale laissant imaginer que la pratique photographique peut figurer au nombre des loisirs ouvriers (ou populaires), parmi d'autres activités sportives ou culturelles. Raymond n'a pas de formation particulière dans ce domaine, il a seulement fréquenté le club photo du comité d'entreprise de l'Aérospatiale. Le journal L'Humanité cependant donnait des instructions précises à ses correspondants dans un fascicule qui paraissait tous les mois : «Des conseils y étaient délivrés [...] Par exemple, une bonne légende devait répondre à cinq questions : Qui Quoi - Quand - Où - Comment. Et c'était un peu pareil pour les photos, il fallait cadrer de telle sorte que la scène puisse être localisée et identifiée grâce à des éléments secondaires (un panneau d'indication, des immeubles, des voitures...) ${ }^{3} . »$. En 1999, L'Humanité supprime ses correspondants locaux, accusés par les photographes titulaires de «manger leur pain ». Raymond n'a pas beaucoup apprécié la façon dont ils ont été traités et aujourd'hui, s'il est toujours adhérent du PCF, il « ne met plus les pieds à L'Huma».

13 À près de 80 ans, Raymond Tessier continue de photographier les manifestations en région parisienne et envoie toujours ses meilleurs clichés à l'URIF CGT, l'Union régionale d'Ile-de-France.

\section{Jacqueline Dubreil, militante du CCFD (entretien réalisé le 26 juin 2014)}

La région nantaise a connu, à la fin des années 1990 et au début des années 2000, une importante mobilisation autour de la question dite des sans-papiers, c'est-à-dire des personnes immigrées, présentes sur le territoire français parfois depuis plusieurs années mais maintenues dans une situation de non-droit par une administration et une législation tantôt indécises, tantôt tatillonnes. Autour d'eux s'est constitué un large collectif de soutien, allant d'organisations chrétiennes (CIMADE et CCFD), à des mouvements d'extrême gauche libertaire, en passant par des militants syndicaux, en particulier de la CGT. L'un d'entre eux, Peter Dontzow, fréquente assidûment le CHT. Ancien membre du secrétariat de l'Union départementale CGT 44, il est notamment connu pour s'être particulièrement investi dans ce mouvement et se souvient avoir réalisé un grand nombre de photographies sur le sujet. Seulement, à ce jour, ses photographies restent introuvables et le CHT ne disposait que de quelques vues relatives à cette lutte, pourtant marquante pour la région nantaise.

De son côté, Jacqueline Dubreil, active au sein de ce collectif de soutien au nom du Comité catholique contre la faim et pour le développement (CCFD), est connue pour avoir réalisé de nombreuses photographies durant tout le mouvement. C'est une autre membre de ce collectif, syndicaliste à la FSU, Emmanuelle Lefèvre, qui prend l'initiative de solliciter Madame Dubreil afin qu'elle autorise le CHT à reproduire ses 
photographies. Suite à ces échanges, nous avons pu réaliser un entretien avec Jacqueline et Alain, son mari, également investi dans le collectif.

16 Madame Dubreil se dit très sensible à la lutte des sans-papiers de Nantes et c'est « instinctivement» qu'elle réalise des photographies, parce qu'elle a conscience de « vivre quelque chose d'exceptionnel ». Si elle est mandatée par le CCFD pour participer au collectif, sa démarche photographique est, au contraire, le fruit de son initiative personnelle. Elle n'en parle pas spécialement aux membres du CCFD dont elle ne photographie d'ailleurs pas les autres aspects du travail quotidien.

Auparavant, elle n'avait jamais réalisé de photos de ses engagements militants et au sein du couple la photo était plutôt le domaine de son mari : «Pendant des années, je n'ai pas touché l'appareil, c'était Alain, mon mari, qui faisait les photos [de famille] ». "C'était un Roleiflex, je ne savais pas m'en servir ». La lutte des sans-papiers coïncide avec l'arrivée d'un nouvel appareil photo (argentique compact) qui lui est offert par ses enfants. À partir du moment où madame Dubreil reçoit son appareil, elle commence à photographier sa famille, à l'inverse son mari se désintéresse de la photo.

Photographie 7. Un sans-papiers au Grenier du siècle.

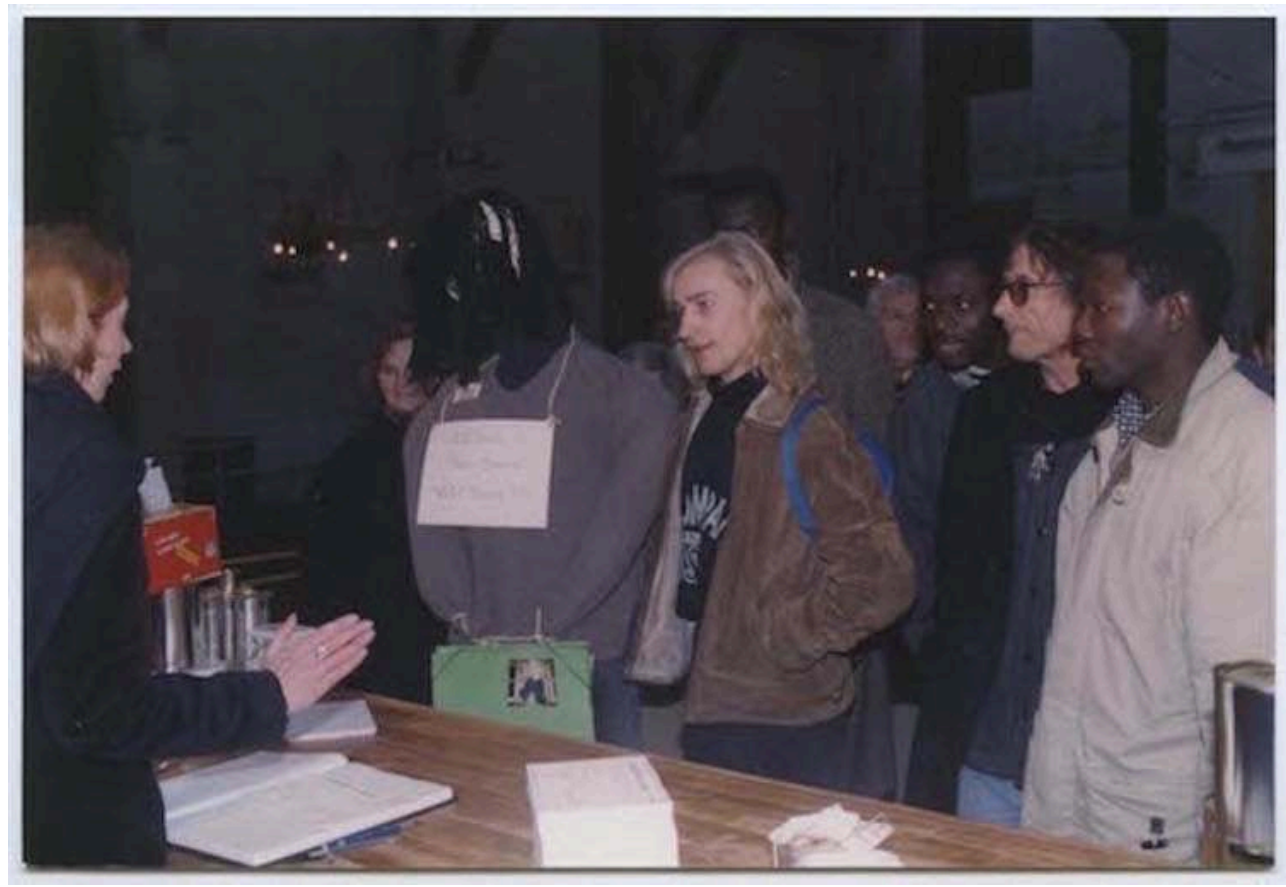

Dépôt d'un mannequin symbolisant un sans-papiers au Grenier du siècle organisé par l'espace LU, à Nantes, novembre 1999

CHT, cliché Jacqueline Dubreil.

18 Jacqueline réalise surtout des portraits et des photos de groupe, «j'aimais bien avoir des gens» et «montrer tous les événements [liés à la lutte]». Elle photographie également les personnalités qu'elle accueille à son domicile pour le compte du CCFD. Elle met toutes ces photos en album, «alors que ça n'est pas le cas pour les photos de famille», dont "beaucoup sont en vrac». À ses yeux, la mise en album est symptomatique de l'importance qu'elle accorde à ces images. Elle le fait au fur et à mesure que les photos sont développées et tirées, c'est un moment de « recueillement » et de « remobilisation». 
19 En dehors de Peter Dontzow, elle ne se souvient pas avoir vu d'autres bénévoles photographier la lutte des sans-papiers. Pourtant, les personnes dont elle tirait le portrait, bénévoles ou sans-papiers, réagissaient très bien, « parfois ils me demandaient un tirage ». "Les gens des autres associations savent que j'ai ces albums et ils me sollicitent de temps en temps ", mais « ça m'a beaucoup étonnée que mes petits albums puissent servir dans un centre comme celui-ci [le $\mathrm{CHT}]$. Je suis très touchée et mon mari en est très fier. J'en ai parlé à mes petits-enfants et ils sont très contents que je dépose ces photos ici ».

\section{Christian Zimmer, un docker nantais (entretien réalisé le 2 juillet 2014)}

Christian Zimmer est un adhérent de longue date du CHT. Docker retraité, il a passé toute sa carrière sur le port de Nantes. À la fois photographe amateur et cartophile, il nous apporte régulièrement des cartes postales en rapport avec l'histoire du port: le travail des dockers bien évidemment, mais aussi la construction navale, le trafic maritime et les conflits sociaux.

21 En 2013, le CHT est associé à un programme de recherche, intitulé « ESCALES ", relatif à l'étude des agents pathogènes auxquels sont exposés les travailleurs portuaires. Afin de populariser les résultats de cette étude, nous décidons de réaliser une exposition sur l'évolution du métier de docker et le caractère de plus en plus insidieux des risques épidémiologiques qui lui sont associés. À cette occasion, Christian Zimmer nous apporte un album de photographies, dont il est en partie l'auteur, sur les dockers de Nantes entre le milieu des années 1980 et le début des années 1990.

Nous avons sélectionné et reproduit 95 images issues de cet album. En effet, contrairement à Jacqueline Dubreil, Christian Zimmer ne classe pas systématiquement ses photos en album, l'essentiel est même stocké dans des boîtes à chaussures sans qu'il $\mathrm{y}$ ait, de son propre aveu, de logique à cette répartition. Nous n'avons donc reproduit qu'une (petite ?) partie de sa collection: «Un jour faudra que je te les montre ». En définitive il ne "montre" pas souvent ses photos, pas même à sa famille ou aux copains : "J'ai dû les sortir deux ou trois fois, et puis après... » Une ou deux sont punaisées au mur, chez lui, dont le portrait de Jean-Luc Chagnolleau, un docker décédé d'un cancer (finalement reconnu d'origine professionnelle par une décision du tribunal de la Sécurité sociale de Nantes en décembre 2014).

Au sein même de l'album, les séries de clichés sont organisées sans logique apparente, pas même chronologique. De plus, nous retrouvons des vues identiques, ou très proches, dans une autre collection conservée par Luc Rousselot ${ }^{4}$, lui aussi docker: "C'est le seul docker [encore en activité sur le port de Nantes] qui fait des photos et qui les garde ». En fait, Luc et Christian échangent leurs clichés, des négatifs puis des fichiers numériques, passent de l'un à l'autre. Chacun réalisant des tirages des vues qui l'intéressent sans se soucier de question de paternité. Ils respectent en cela une forme de propriété collective, courante dans bien des communautés de travail.

Christian est ouvert à l'échange avec des collectionneurs passionnés de la vie du port, pas seulement des dockers. Il lui arrive de passer des annonces en ce sens dans la presse locale. Ses photos ne sont donc pas interdites d'accès, il fait peut-être simplement preuve d'une certaine forme de réserve ou de pudeur qui le poussent à ne pas 
« exhiber » ses documents en permanence auprès de ses proches. Lorsque le CHT le lui a demandé, il n'a pas hésité à autoriser leur reproduction : "Vous êtes les premiers à me solliciter ». " Je suis content, si quelqu'un a besoin de faire un travail, tu as toutes mes données, ma carte de pointage, une carte " $G$ " [le sésame longtemps indispensable pour travailler sur les quais] ». Car l'album de Christian ne contient pas seulement des photos, mais aussi une partie de ses documents professionnels.

"On a eu l'instinct de faire des photos». C'est en ces termes que Christian Zimmer explique le début de sa démarche photographique sur les quais. Il commence au milieu des années 1980, à peu près en même temps que Luc, mais il ne se souvient pas d'un élément déclencheur : «Le dommage c'est qu'on ne l'a pas fait plus vite [...] Quand on voit tous les copains qui sont partis». Rétrospectivement, il a conscience que cette démarche a permis de « constituer un fonds [documentaire]».

Photographie 8. À fond de cale.

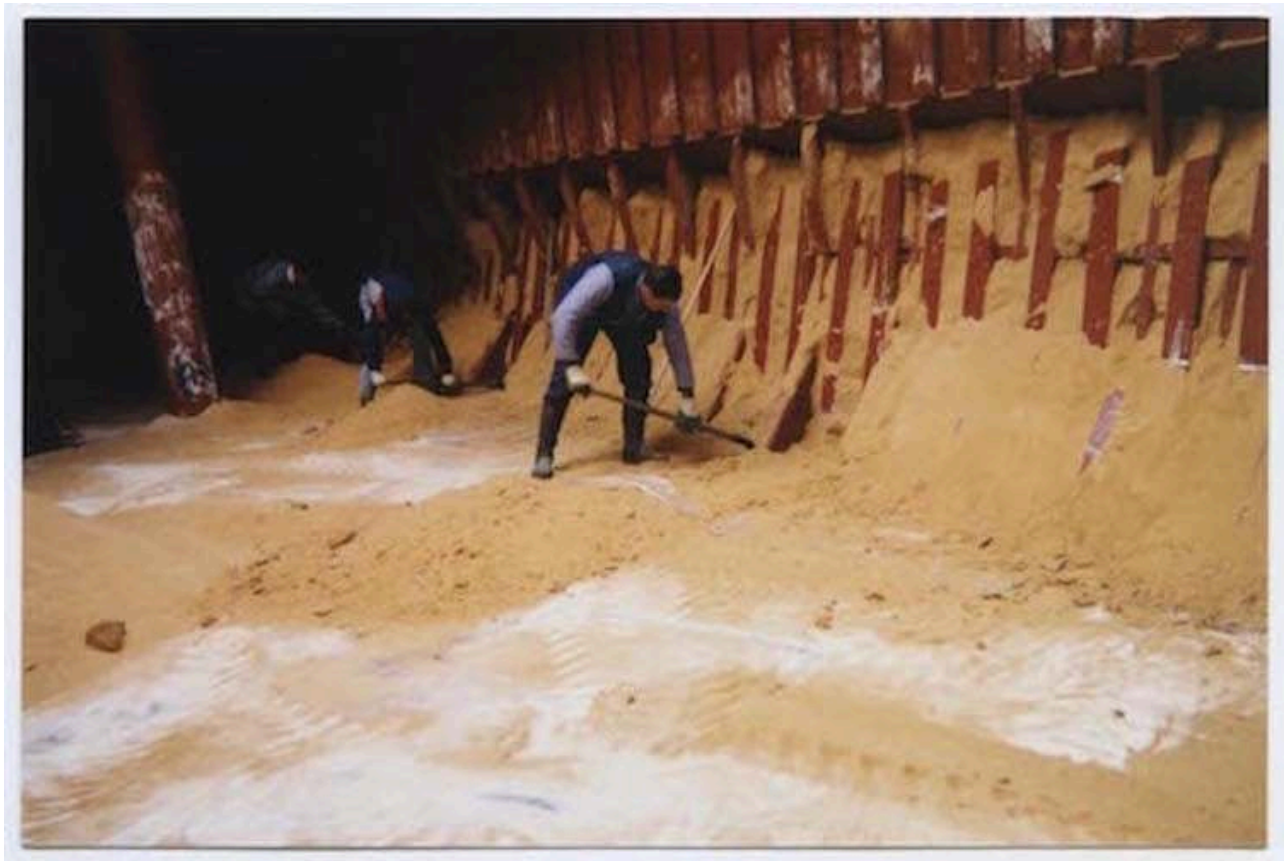

Déchargement d'un bateau de sucre, quai de Cheviré dans le port de Nantes : travail de finition et de nettoyage en fond de cale, non daté (années 2000).

$\mathrm{CHT}$, cliché Christian Zimmer.

Sur le plan familial ou privé, son activité photographique commence quelques années plus tôt. En particulier en 1977, date à laquelle il achète un appareil reflex, argentique, et s'inscrit dans un club photo de quartier, à Orvault. «Je voulais apprendre les bases. C'était intéressant, on faisait des prises de vue et après on pouvait développer. » Mais « ils ne s'occupaient pas assez des nouveaux arrivants. Moi, je ne fonctionne pas comme ça! Au sport [Christian est un judoka confirmé], quand il y a un débutant, on va l'aider...»

Lorsqu'il commence à photographier le travail des dockers, Christian Zimmer s'équipe d'un appareil compact qu'il glisse dans sa poche à l'embauche. Il doit prévenir ses collègues qu'il a l'intention de prendre des photos pendant le service, mais il ne peut pas les arrêter en plein effort. Encore une fois, « c'est à l'instinct ». « Un copain docker a pris une photo sans prévenir les gars, il s'est fait engueuler [Christian et Luc ne sont 
donc pas tout à fait seuls à pratiquer l'auto-représentation]». Alors pensez-donc s'il s'agit d'un photographe extérieur: "les gars n'aiment pas être pris en photo". Pourtant, particularité du monde des dockers, il n'y a pas (il n'y avait pas à l'époque) de hiérarchie pour interdire ces prises de vue.

Aujourd'hui à la retraite, Christian a cessé de réaliser des photos du travail sur le port, " comme Luc [qui est encore en activité] a toujours son appareil», "et puis ça va devenir de plus en plus dur d'entrer sur les quais avec les nouvelles normes... »

\section{Maryse Choquet et les « filles de Chantelle » (entretien réalisé le 6 novembre 2014)}

Maryse Choquet a été l'une des responsables de la section syndicale CFDT de l'usine Chantelle à Saint-Herblain. Elle entre dans cette entreprise de confection de lingerie en 1972, comme simple ouvrière, et la quitte à la fermeture du site herblinois, en 1995. Beaucoup d'habitants de la région nantaise associent cette usine à deux conflits mémorables, l'un en 1981, l'autre pendant l'hiver 1993-1994. Les « Filles de Chantelle ", comme on les appelle souvent, gagnent le respect de tous du fait de leur ténacité et offrent un exemple de combativité syndicale dans un secteur fortement féminisé, rarement très syndiqué et indépendant des traditionnels bastions ouvriers de la métallurgie. Depuis quelques années, un regain d'intérêt des scientifiques pour l'histoire ouvrière, notamment en sociologie, a conduit plusieurs d'entre eux à s'intéresser à cette entreprise et aux conflits dont elle a été le théâtre. Deux thèses ont été produites (Gallot, 2013 et Meuret-Campfort, 2014) ainsi qu'un web documentaire ${ }^{5}$, pour lesquels le $\mathrm{CHT}$ a fortement été sollicité.

Ces travaux furent l'occasion de mettre à jour de nouvelles archives, et en particulier deux albums de photographies. L'un de ces albums, contenant 32 photos, est conservé dans les locaux de l'Union locale CFDT de Nantes dont Maryse est aujourd'hui salariée. Cet album n'est pas le premier : « On en avait fait un en 1976 [lors d'un premier conflit], mais nous n'avions pas trop de pratique. En 1981, nous nous sommes dit : "Un conflit ça s'exploite”, et puis les photos ça reste. [...] En 1993, c'était pareil, il y avait une personne qui était responsable de la presse : elle faisait les photos, elle s'occupait de la presse, elle découpait les articles de presse, elle mettait ça dans un album. " Donc dès les années 1970, une section syndicale d'une "petite » entreprise textile réfléchit à son image et prend l'initiative de photographier ses luttes.

Il s'agit d'une démarche préméditée et réfléchie collectivement. Cette tâche est dévolue à une militante (le personnel de l'entreprise est très majoritairement féminin) qui n'a pas (encore) de responsabilité syndicale importante et qui, pendant le conflit, n'est pas accaparée par la conduite de la lutte ou la participation aux négociations. En 1976, Maryse hérite de cette fonction: «Je n'avais pas de mandat syndical, j'étais disponible pour ça. Après, en 1981, ça a été un peu plus compliqué parce que j'avais des mandats syndicaux. Après [en 1993] ça a été confié à quelqu'un d'autre. »

Rétrospectivement, il est facile de considérer que tel ou tel conflit mérite d'être photographié et documenté mais, dans le feu de l'action, comment ces militantes s'organisent-elles? À quel moment décident-elles de prendre des photos? «Pour les simples débrayages on ne prenait pas l'appareil photo. Là [en déc. 1993], on avait senti que ça allait être quelque chose de fort. Donc on avait dit "je crois qu'il va nous le 
falloir". D'ailleurs, la preuve, dès le premier jour, on avait l'appareil photo. » En fait, au sein de la section CFDT Chantelle, l'appareil photo fait partie du matériel syndical, au même titre que le mégaphone.

Pendant le conflit de 1993, les photos sont mises en album au fur et à mesure de leur tirage, avec des annotations et des légendes. C'est un travail de la section toute entière, "mais on n'avait pas le temps de se dire "qu'est-ce qu'on va faire de ces photos". On était dedans pendant un an [c'est la durée du conflit de 1993-1994]. » De fait, les photos ne sont pas publiées, pas même dans les journaux syndicaux, et les tracts de l'époque ne sont pas illustrés. En revanche, dans le local syndical, il y avait un panneau d'affichage où étaient exposées certaines photos, prises par des militantes de la section ou des journalistes comme Hélène Cayeux.

En dehors de cette démarche syndicale, Maryse Choquet ne se souvient pas avoir vu d'autre gréviste prendre des photos: "Les filles savaient qu'on allait prendre des photos, mais je n'ai pas le souvenir de voir d'autres personnes avec un appareil photo. » Quelques ouvrières demandaient au syndicat de leur retirer certaines photos, «mais ce n'était pas une majorité. » Pourtant, "elles étaient très, très contentes d'être sur les photos. Jamais je n'ai entendu : “Ah non, je ne veux pas être sur la photo.” ”

Cette démarche syndicale novatrice débouche-t-elle pour autant sur une prise de conscience photographique plus globale et une volonté, chez ces ouvrières, de s'emparer de leur image sur leur lieu de travail ? Au sein de l'usine, « on ne faisait pas de photos en dehors des conflits [...] À part à Noël où on faisait un petit peu les andouilles. " La direction s'efforce de maîtriser au maximum l'image de l'entreprise. "Je ne sais plus si c'était le conflit de 1993 ou un autre, à chaque fois qu'on demandait l'autorisation de faire entrer un journaliste, on avait un refus systématique. Donc au bout d'un moment on a arrêté de leur demander, c'était plus simple. » Selon Maryse, il ne s'agit pas tant d'une question d'espionnage industriel que d'une volonté de la direction de dire: "C'est notre propriété, on n'a pas à ouvrir les portes à qui que ce soit, [...] on est chez nous!» 
Photographie 9. Des chaînes et des cadenas pour sauver Chantelle.

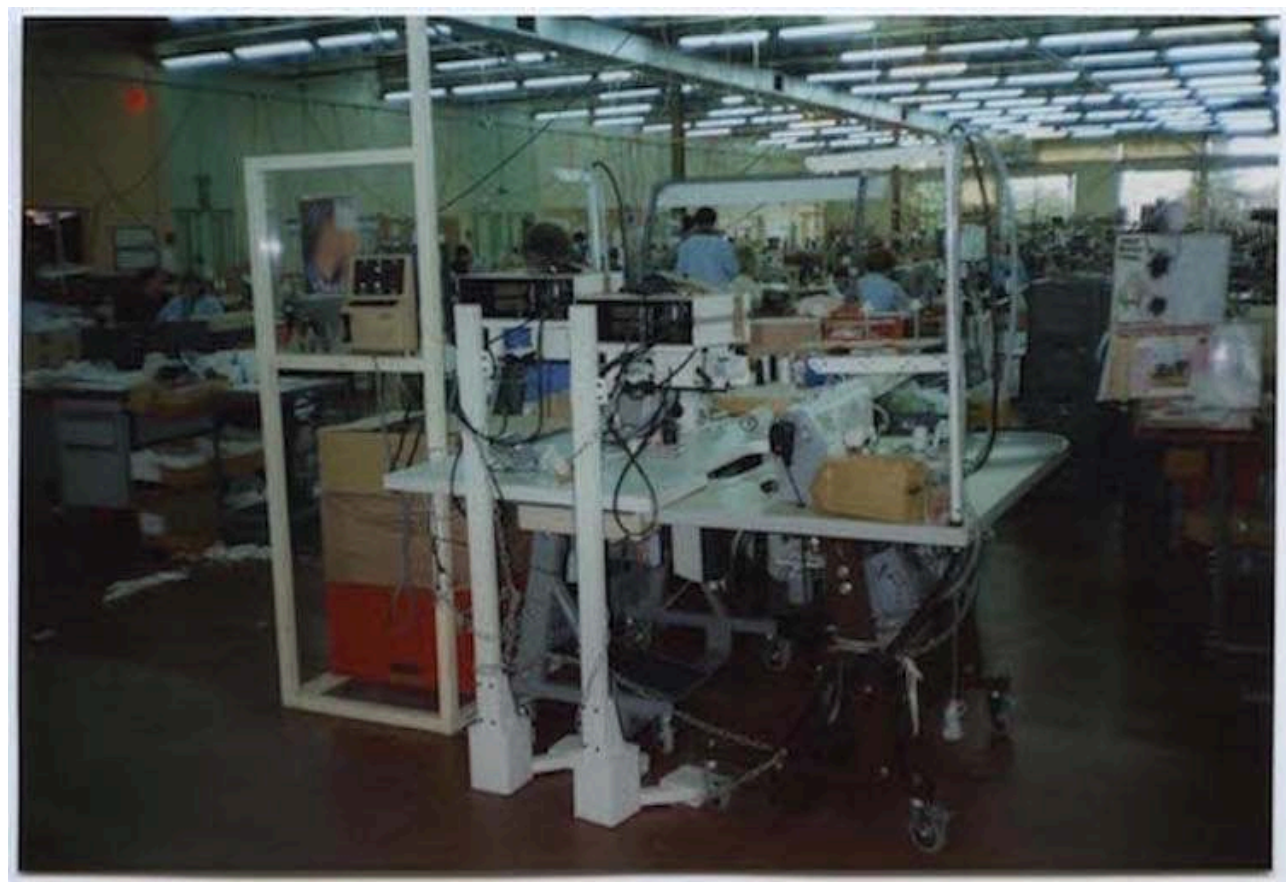

Des chaînes et des cadenas sont posés par les ouvrières « pour empêcher la direction d'enlever des machines ». Cliché réalisé à l'intérieur des ateliers en octobre 1994.

CHT, coll. UL CFDT Nantes. (parfois longues) où la direction perd le contrôle de l'image de l'entreprise. Dans le cas qui nous intéresse, la production n'étant pas complètement arrêtée, les ouvrières ont l'idée de se représenter à leur poste de travail. Cela produit des images ambiguës où, si l'observateur n'a pas d'explications, il a l'impression d'avoir à faire à des non-grévistes. En 1981 déjà, les grévistes ont photographié l'ensemble du processus productif, poste par poste ${ }^{6}$. À notre connaissance, ces photos produites en 1981 ou en 1993-1994 sont les seules disponibles sur le travail dans cette usine.

Maryse, à titre personnel, est évidemment sensible à la photographie. Elle est photographe amateur mais elle n'a jamais fait partie d'un club photo. Elle a été initiée par l'un des membres de sa famille qui lui a « appris à bien cadrer». Elle ne tarde pas à remarquer le travail d'Hélène Cayeux, une photographe de l'AFP, puis du journal OuestFrance, qui couvre aussi bien le conflit de 1981 que celui de 1993. Hélène Cayeux est réputée auprès des militants syndicaux, toutes tendances confondues, pour être capable de s'immerger au cœur des conflits sociaux et de prendre le temps de réaliser des clichés qui deviennent souvent emblématiques de ces luttes. La grève "des Chantelle " n'échappe pas à son objectif et, comme à son habitude, elle distribue généreusement ses clichés. «Elle nous avait carrément prêté ses négatifs [...] On est allé la voir dans son bureau, à Ouest-France, et elle nous les avait prêtés pour qu'on en fasse des retirages. » Maryse se souvient même de discussions avec Hélène Cayeux sur la photographie : "Quelle ouverture choisir », «Comment centrer une photo »... «C'est marrant, je me dis que j'aurais pu faire quelque chose de sympa dans la photographie. » 
Par la suite Maryse a suivi le travail d'Hélène Cayeux. Elle est allée visiter ses expositions, par exemple Images de boites à la Maison des hommes et des techniques, une structure créée par les anciens métallos de la Navale nantaise.

\section{Conclusion : une histoire à écrire}

Ces quelques entretiens nous incitent à penser qu'une histoire populaire de la photographie reste à écrire : l'aventure des clubs photo, la pratique intuitive de l'autoreprésentation, ces clichés réalisés par les salariés sur leur lieu de travail et de leur propre initiative, l'existence de familles de photographes ouvriers, l'usage d'albums pour conserver des documents (et pas seulement des photographies) relatifs à son univers professionnel, nous laissent entrevoir un nombre considérable de champs à explorer. L'observation de la pénétration de la photographie au sein du monde du travail, et des classes populaires en général, met en évidence des enjeux de pouvoir au sein de l'entreprise, dotés d'une forte dimension symbolique: qui a le droit de représenter le monde du travail? Qui exerce véritablement ce droit? Comment se représenter une entreprise (ou une profession) qui n'existe plus? Telles sont quelquesunes des questions qu'il ne faudrait pas omettre de se poser.

Enfin, du fait des lacunes, des manques d'images constatées dans bien des domaines, nous pouvons dire que l'intérêt d'une photographie du monde du travail, pour toute personne désireuse d'observer cet univers, qu'elle soit chercheuse ou militante, dépend autant de l'intention du photographe que de l'usage qui en est fait ultérieurement et de la réception de celle-ci par ses observateurs. La photographie est un tout qui ne se restreint pas à la seule captation de la lumière, et l'histoire d'un cliché n'est jamais close tant son interprétation est tributaire des circonstances et donc des enjeux du moment.

\section{BIBLIOGRAPHIE}

Nerrière X. (2014), Images du travail - Les collections du Centre d'histoire du travail de Nantes, PUR.

Gallot F. (2013), Les ouvrières, des années 1968 au très contemporain : pratiques et représentations, thèse de doctorat, université Lumière Lyon 2, publiée aux éditions de la Découverte sous le titre En découdre-Comment les ouvrières ont révolutionné le travail et la société.

Meuret-Campfort E. (2014), Des ouvrières en lutte - Mondes populaires et genre du syndicalisme dans un secteur d'emploi «féminin »-Le cas de l'usine Chantelle à Nantes (1966-2005), thèse de doctorat, université Nantes Angers Le Mans.

\section{Webdocumentaire}

Marchand A. et Lejosne A-L. (2014), Les dessous de la fabrique, Fil en têtes, [En ligne]. URL : http:// www.lesdessousdelafabrique.fr/ 


\section{NOTES}

1. Le Centre d'histoire du travail est tout à la fois un centre d'archives, un centre de documentation, une bibliothèque et une maison d'édition consacrés aux mouvements sociaux à l'échelle de la Loire-Atlantique. Il est créé sous forme associative en 1981, à l'initiative d'universitaires et avec le soutien des principales organisations syndicales: les unions départementales (UD) CGT, CFDT, CGT-FO et FEN puis la FDSEA, récemment rejointes par la FSU (www.cht-nantes.org/opacce/).

2. Soulignons, dans ce domaine, le travail du Centre de culture populaire de Saint-Nazaire (www.ccp-asso.org/), un inter-CE, qui, dans le but de faire entrer l'art dans les entreprises, accueille régulièrement des photographes en résidence.

3. L'organisation du journal l'Humanité rappelle celle du journal Regard sur le monde du travail, une publication également liée au PCF qui, dans les années 1930, s'appuyait sur les Amateurs photographes ouvriers (APO). Nous ne connaissons cependant pas d'étude sur le sujet et nous remercions Dominique Versavel de la BNF qui a attiré notre attention sur un article paru dans ce journal : Regard sur le monde ouvrier, n³, mars 1932.

4. Un entretien identique est prévu avec Luc Rousselot.

5. Aurélie Marchand et Anne-Laure Lejosne, Les dessous de la fabrique, Fil en tête, 2014. Web documentaire consultable en ligne : http://www.lesdessousdelafabrique.fr/

6. Ces images sont conservées par Pascale Bourhis, une militante CGT qui a également laissé le CHT reproduire ses albums.

\section{RÉSUMÉS}

Après de nombreuses années passées à classer et indexer des photographies issues d'archives syndicales, Xavier Nerrière essaie de mettre en évidence l'intérêt, tant scientifique que patrimonial, de la notion d'auto-représentation. Ces images, produites par les salariés euxmêmes, sur leurs lieux de travail (en situation de travail ou lors de conflits sociaux) et de leur propre initiative, constituent un objet d'étude complexe qui doit être accompagné de métadonnées: qui a pris ces clichés, quand, pourquoi, à quoi ont-ils servi ? Avec de telles informations, les perspectives de questionnement et de recherche sont démultipliées. L'auteur présente quatre cas concrets de militants syndicaux, politiques ou associatifs (les problématiques étant très proches), qui ont accepté de confier une partie de leur production photographique au Centre d'histoire du travail de Nantes et de préciser leurs motivations.

After many years spent sorting and indexing photographs from labour union archives, Xavier Nerrière aims at bringing out the interest of the concept of self-representation, in the scientific as well as in the patrimonial field. These pictures by the workers, in their workplaces (be it at work or during social conflicts) and their own, represent a complex subject of study which has to be accompanied by metadata: who took these pictures, when, in what circumstances, why, what for? With such information, the prospects of questioning and of research are compounded. The author develops four specific cases of labour activists, either from the political or from the associative sector (the issues being quite similar). These four people have accepted to leave some of their photographical data to the Institute of Labour History of Nantes (Centre d'histoire du travail de Nantes) and specify their motivations. 


\section{INDEX}

Mots-clés : photographie amateur, auto-représentation, culture ouvrière, classe ouvrière, patrimoine, luttes sociales, monde du travail, syndicalisme

Keywords : amateur photography, self representation, working class culture, working class, heritage, social struggles, world of work, labour unionism

\section{AUTEUR}

\section{XAVIER NERRIÈRE}

Xavier Nerrière est animateur-chercheur au sein du Centre d'histoire du travail de Nantes (CHT), une association soutenue par les principales organisations syndicales de salariés de LoireAtlantique, qui a pour objet la collecte, la conservation et la valorisation de la mémoire des luttes sociales dans le département. Juriste de formation, l'auteur gère depuis plus de quinze ans les fonds iconographiques déposés au CHT. Après plusieurs articles consacrés à la photographie patrimoniale dans des revues comme 303, Place publique, des chroniques régulières dans ADEN, une revue littéraire consacrée aux années 1930, il a fait part de ses réflexions et de son expérience dans un ouvrage de synthèse intitulé Images du travail - Les collections du Centre d'histoire du travail de Nantes, publié aux PUR en 2014 (voir la présentation dans la revue ITTI n 1 , de janvier 2016). Il est également intervenu lors d'un colloque organisé par l'université de Namur, en mars 2014, intitulé : Quand l'image (dé)mobilise - Iconographie et mouvements sociaux au $\mathrm{XX}^{e}$ siècle (dont les actes ont été publiés aux Presses universitaires de Namur en 2015). 